\title{
PAIRWISE COLLIDING PERMUTATIONS AND THE CAPACITY OF INFINITE GRAPHS
}

\author{
JÁNOS KÖRNER AND CLAUDIA MALVENUTO
}

\begin{abstract}
We call two permutations of the first $n$ naturals colliding if they map at least one number to consecutive naturals. We give bounds for the exponential asymptotics of the largest cardinality of any set of pairwise colliding permutations of $[n]$. We relate this problem to the determination of the Shannon capacity of an infinite graph and initiate the study of analogous problems for infinite graphs with finite chromatic number.
\end{abstract}

\section{INTRODUCTION}

Let $n$ be an arbitrary natural number and let $[n]$ be the set of all natural numbers from 1 to $n$. We will say that two permutations of $[n]$ are colliding if they map at least one element of $[n]$ into two consecutive numbers, i.e. into numbers differing by 1. It is then natural to ask for the determination of the maximum cardinality $\rho(n)$ of a set of pairwise colliding permutations of $[n]$. One easily sees that this number grows exponentially with $n$ and its asymptotic exponent lies between $\log _{2} \frac{1+\sqrt{5}}{2}$ and 1. We will prove this and some better bounds later on.

Certain graphs having as vertex set the permutations of $[n]$ have been introduced before by Cameron and $\mathrm{Ku}$ [1] and Larose and Malvenuto [10], cf. also $\mathrm{Ku}$ and Leader [9] for a generalization. These authors considered Kneser-type graphs in which they studied the growth of stable sets describing sets of permutations that are "similar" in some sense, whereas our definition of adjacency corresponds to being "different" and distinguishable in some other, particular sense. In fact, the above Kneser-type problems, unlike ours, have no immediate relation to capacity in the Shannon sense.

In this paper we will generalize our introductory problem in several ways. We will consider arbitrary infinite graphs over the natural numbers and introduce various new concepts of capacity. As always, graph capacity measures the exponential growth rate of the largest cliques induced on the Cartesian powers of the vertex set of a graph. In case of an infinite vertex set such as the naturals this is not always interesting, for the graph in itself might have infinite cliques. Then it is reasonable to restrict our attention to particular subsets of the power sets, e. g. those representing permutations. We will present some simple bounds for the value of the so obtained new capacities.

\section{PERMUTATION CAPACITY}

Let $G$ be an arbitrary graph with a countable set of vertices. Without loss of generality we can suppose that the vertex set $V(G)$ of $G$ is the set $\mathbb{N}$ of natural numbers. Further, let us denote by $G[A]$ the subgraph of $G$ induced by an arbitrary subset $A$ of the vertex set of $G$. As usual, we also consider, for every natural 
$n \in \mathbb{N}$, the power graph $G^{n}$ whose vertex set is $\mathbb{N}^{n}$, the set of $n$-length sequences of natural numbers. Two such sequences $\mathbf{x} \in \mathbb{N}^{n}$ and $\mathbf{y} \in \mathbb{N}^{n}$ are adjacent in $G^{n}$ if $\mathbf{x}=x_{1} x_{2} \ldots x_{n}$ and $\mathbf{y}=y_{1} y_{2} \ldots y_{n}$ have at least one coordinate $i \in[n]$ for which $\left\{x_{i}, y_{i}\right\} \in E(G)$, i.e. if the vertices $x_{i}$ and $y_{i}$ are adjacent in $G$. (This concept of power graph is rooted in information theory. If we interpret adjacency of vertices of a graph as a relation of distinguishability, it is very intuitive to extend such a notion to strings of vertices in the above way, with the meaning that two strings are distinguishable if we can distinguish them in at least one of their coordinates.)

Throughout this paper we write $\left(\begin{array}{l}X \\ n\end{array}\right)$ for the family of all $n$-element subsets of $X$. For an arbitrary set $A \in\left(\begin{array}{l}\mathbb{N} \\ n\end{array}\right)$ we write $R(A)$ for the set of all the $n$-length sequences without repetitions on the alphabet $A$. As usual, we can think of a sequence in $R(A)$ as a permutation of the set $A$. In particular, when $A=[n]$, the sequence $\mathbf{x}=x_{1} \ldots x_{n} \in R([n])$ represents the permutation of $[n]$ which maps $i$ into $x_{i}$.

We denote by $G(A)$ the subgraph of the power $G^{n}$ induced by $R(A)$ and by $\rho(G, A)$ its clique number. We set $\rho(G, n)$ for the largest cardinality of a clique induced by $G^{n}$ on the sequences corresponding to the permutations of an $n$-set in $V(G)$, i.e.

$$
\rho(G, n)=\max _{A \in\left(\begin{array}{c}
\mathbb{N} \\
n
\end{array}\right)} \rho(G, A)
$$

Finally, we define

$$
\rho(G)=\limsup _{n \rightarrow \infty} \frac{1}{n} \log _{2} \rho(G, n)
$$

and call it the permutation capacity of the graph $G$.

In this paper we consider some infinite graphs and try to determine their permutation capacity. Since our graphs have a countable set of vertices, the value of their permutation capacity might well be infinite. The same is true for Shannon capacity. In fact, to our knowledge Shannon capacity of infinite graphs has not been considered so far, even though it makes perfect sense and will be discussed below.

The problem of the asymptotic growth of cliques of particular induced subgraphs of $G^{n}$ as $n$ goes to infinity, is a key ingredient in determining the Shannon capacity of graph families in the sense of Cohen, Körner and Simonyi [2], where the sets inducing the subgraphs are formed by all the sequences "of a given type", in an information theoretic sense (see Csiszár and Körner [3] for a definition and more on this). All the sequences of a given "type" form a minimal set that is invariant under the action of all the permutations of the coordinates of the sequences. Our present concepts are natural extensions to the case of infinite graphs of Shannon capacity in a given type, in the sense of [3].

\section{Examples}

Let us start with an atypical and even somewhat trivial example, just to rephrase the already cited results of [1] and [10] in our present terms.

Consider the graph $G$, where $V(G)=\mathbb{N}$ and $E(G)=\{\{x, x\}: x \in \mathbb{N}\}$, consisting of loops on the natural numbers. When $A=[n]$, its set $R(A)$ is the set of permutations of $[n]$ and two permutations $\mathbf{x}=x_{1} \ldots x_{n}$ and $\mathbf{y}=y_{1} \ldots y_{n}$ are adjacent if and only if there is a coordinate $i \in[n]$ such that $x_{i}=y_{i}$. We will denote by $\mathbf{x}^{-1}$ the inverse of the permutation represented by the sequence $\mathbf{x}$. With this notation, $\mathbf{x}$ and $\mathbf{y}$ are adjacent if the product $\mathbf{x y}^{-1}$ is not a derangement. This is the complement 
of the graph of permutations studied by Cameron-Ku [1] and Larose-Malvenuto [10], that is the Cayley graph of permutations with generators the derangements. It is obvious that $\rho(G, n)=\rho(G,[n])=(n-1)$ ! and thus the clique number $(n-1)$ ! is super-exponential in $n$. In fact, the above authors show far more than this; they prove that the trivial construction, consisting of the set of all permutations that map an arbitrary fixed natural $l$ into an arbitrary fixed natural $m$, is the unique way to achieve the clique number. This graph is somewhat artificial in the present context. If in a graph the only edges are loops, then adjacency corresponds to "being similar". In graph capacity problems one usually considers only graphs without loops and interprets adjacency as some sort of distinguishability between vertices. From now on we will restrict attention to these cases.

One of the simplest and perhaps most natural examples of our present problem is furnished by the (semi-)infinite path $L$ whose vertices $x$ and $y$ from $\mathbb{N}$ are adjacent if they are consecutive in the natural order, that is $|y-x|=1$. Clearly, $\omega(L)=\chi(L)=$ 2 and thus the Shannon capacity $\log \lim _{n \rightarrow \infty n} \sqrt[n]{\omega\left(L^{n}\right)}$ equals 1 (cf. Shannon [13], Lovász [11] and in particular Cohen, Körner and Simonyi [2], where the problem is reformulated, geared towards the subsequent generalizations [6] and[7], in the present terms ). We will show that

$$
\log _{2} \frac{1+\sqrt{5}}{2} \leq \rho(L) \leq 1
$$

For the infinite path $L$, denote simply by $L(n)$ rather than $L([n])$ the subgraph induced by the set $A=[n]$ on the $n$-th power of $L$ : its vertex set is the set of all the permutations of the set $[n]$ (the permutations of $n$ elements) and two of such permutations $\mathbf{x}=x_{1} \ldots x_{n}$ and $\mathbf{y}=y_{1} \ldots y_{n}$ are adjacent in $L(n)$ if and only if the following condition holds:

$$
\exists i \in[n]:\left|y_{i}-x_{i}\right|=1 .
$$

Note that, as observed in [5], every finite graph is an induced subgraph of $L^{n}$ for some value of $n$.

The two graphs above belong to a more general class of graphs $G(\mathcal{D})$ depending on a finite subset $\mathcal{D}$ of $\mathbb{N}$ of "allowed differences" as follows: its vertices are, as before, the natural numbers $\mathbb{N}$ and $\{x, y\} \in G(\mathcal{D})$ if and only if $|x-y| \in \mathcal{D}$. When $\mathcal{D}=\{0\}$ we have the all-loops graph described above; when $\mathcal{D}=\{1\}$ we have $G(\mathcal{D})=L$.

\section{The InFINITE PATH}

In this section we will study the behavior of the cliques in the powers of the (semi-)infinite path $L$. In particular, we will derive some recursive inequalities for the value of $\rho(L, n)$.

Observation. For any $n$-element subset $A$ of the naturals the graph induced on it by $L$ is isomorphic to a subgraph of the path of $n$ vertices induced by $L$ on the set $[n]$. Hence by an obvious monotonicity

$$
\rho(L, n)=\max _{A \in\left(\begin{array}{l}
\mathbb{N} \\
n
\end{array}\right)} \rho(L, A)=\rho(L,[n]) .
$$

In other words, $\rho(L, n)$ is the maximum number of permutations of $[n]$ such that for any two of them, there is an element of $[n]$ mapped into two consecutive integers 
from $[n]$. Recall that this is the very same problem we introduced at the beginning of this paper where we wrote $\rho(n)$ for $\rho(L, n)$.

The following recursive inequality will play a key role in our attempt to determine the permutation capacity of the infinite path.

Proposition 4.1. The function $\rho(L, n)$ is super-multiplicative:

$$
\rho(L, n+m) \geq \rho(L, n) \cdot \rho(L, m) .
$$

Proof. Take a clique $C$ in $L(n)$ of maximal size $\rho(L, n)$ and a clique $D$ of maximal size $\rho(L, m)$ in $L(m)$. Denote by $D+n$ the set obtained from $D$ by adding $n$ to each element of the sequences of $D$ :

$$
D+n=\left\{x_{1}+n \ldots x_{m}+n: x_{1} \ldots x_{m} \in D\right\} \subseteq R(\{n+1, \ldots, n+m\}) .
$$

Clearly the size of the clique $D+n$ in $G(\{n+1, \ldots, n+m\})$ is the same as that of $D$. Hence the product construction

$C \times(D+n)=\left\{x_{1} \ldots x_{n+m}: x_{1} \ldots x_{n} \in C ; x_{n+1} \ldots x_{n+m} \in D+n\right\} \subseteq R([n+m])$,

obtained by concatenating sequences from $C$ to sequences from $D+n$, gives a clique in $G(n+m)$ of size $\rho(L, n) \cdot \rho(L, m)$.

By the well-known elementary inequality called Fekete's lemma (see [14]), the last proposition implies that the $\operatorname{limit}_{\lim } \rightarrow \infty \sqrt[n]{\rho(L, n)}$ exists, and its logarithm coincides with the permutation capacity $\rho(L)$.

It is immediately obvious that the capacity $\rho(L)$ is upper bounded by the logarithm of the chromatic number of $L$, and thus is at most 1 . The following nonasymptotic refinement might be interesting.

\section{Proposition 4.2.}

$$
\rho(L, n) \leq\left(\begin{array}{c}
n \\
\left\lfloor\frac{n}{2}\right\rfloor
\end{array}\right) .
$$

Proof. Call parity pattern of a permutation $\mathbf{x}=x_{1} x_{2} \ldots x_{n}$ the binary sequence of length $n$ obtained when substituting every entry in $\mathbf{x}$ by its congruence class modulo 2. Now observe that if two permutations $\mathbf{x}$ and $\mathbf{y}$ are colliding, which means that there is a coordinate $i$ such that $x_{i}$ and $y_{i}$ are consecutive integers, then in the $i$-th coordinate of the corresponding parity patterns there is a difference in 0 and 1, implying that their parity patterns are different: so in a clique of $L(n)$ there is at most one permutation for any given parity pattern. Finally, the parity pattern of a permutation of $[n]$ has $\left\lfloor\frac{n}{2}\right\rfloor$ 's and $\left\lceil\frac{n}{2}\right\rceil$ 1's.

\section{Proposition 4.3.}

$$
\rho(L, n) \geq \rho(L, n-1)+\rho(L, n-2) .
$$

Proof. Take a clique $C$ of maximal size for $L(n-1)$ and a clique $D$ of maximal size for $L(n-2)$. Now set $\hat{C}:=\left\{x_{1} \ldots x_{n-1} n: x_{1} \ldots x_{n-1} \in C\right\}$ and $\hat{D}:=$ $\left\{x_{1} \ldots x_{n-2} n(n-1): x_{1} \ldots x_{n-2} \in D\right\}$. In this way any element from $\hat{C}$ will collide with any element from $\hat{D}$ in the last coordinate because of the edge $\{n, n-1\}$, so that $\hat{C} \cup \hat{D}$ is a clique in $L(n)$ of size $\rho(L, n-1)+\rho(L, n-2)$. 


\section{Corollary 4.4.}

$$
\log _{2}\left(\frac{1+\sqrt{5}}{2}\right) \leq \rho(L) \leq 1 .
$$

Proof. Although the present upper bound to the permutation capacity of the infinite path is obvious as observed before, for the sake of completeness we deduce from Proposition 4.2 that $\frac{1}{n} \log _{2} \rho(L, n) \leq \frac{1}{n} \log _{2}\left(\begin{array}{c}n \\ \left\lfloor\frac{n}{2}\right\rfloor\end{array}\right) \leq 1$.

For the lower bound, Proposition 4.3 shows, together with $\rho(L, 1)=1$ and $\rho(L, 2)=2$, that the sequence $\rho(L, n)$ grows at least as fast as the basic Fibonacci sequence $F(n)$ : since $\lim _{n \rightarrow \infty} \sqrt[n]{F(n)}=\frac{1+\sqrt{5}}{2}$, we get $\log _{2}\left(\frac{1+\sqrt{5}}{2}\right) \leq \rho(L)$.

A non-recursive way of constructing a clique of size $F(n)$ in $L(n)$ is the following. Consider the set $S$ of permutations obtained from the identical permutation by exchanging two consecutive integers, i.e. $S=\left\{s_{i}: i=1, \ldots, n-1\right\}$ where $s_{i}=$ $(i, i+1)$ is the adjacent transposition, in cyclic notation. For $I=\left\{i_{1}<\ldots<i_{k}\right\} \subseteq$ $[n-1]$ let $s_{I}=s_{i_{1}} \ldots s_{i_{k}}$. Let

$$
C(n)=\left\{J \subseteq[n-1]: \forall i, j \in J s_{i} s_{j}=s_{j} s_{i}\right\}
$$

be the family of subsets of $[n-1]$ whose corresponding adjacent transpositions are pairwise commuting. Since for $i \neq j$ one has $s_{i} s_{j}=s_{j} s_{i}$ if and only if $|i-j| \geq 2$, we can encode the elements of $C(n)$ as zero-one sequences of length $n-1$ with the property that no consecutive 1's appear in the sequence. Since the number of zero-one sequences of length $n$ without consecutive "1"'s is known to be $F(n)$, and since each of these is in bijection with some element of $C(n)$, we see that in $C(n)$ there are exactly $F(n)$ sequences. Furthermore for $I, J \in C(n)$ with $I \neq J$ one has $\left\{s_{I}, s_{J}\right\} \in E(L(n))$ : let $h=\min I \Delta J$, where $\Delta$ denotes the symmetric difference of sets, and suppose that $h \in I$; then clearly $h \notin J, h+1 \notin I$ because of the condition on $C(n)$ and $h-1 \notin J$ by the minimality of $h$ : whence we deduce that $s_{I}(h)=h+1$ and $s_{J}(h)=h$, that is $s_{I}$ and $s_{J}$ are adjacent.

For $n=4$, the set of binary sequences $\{000,100,010,001,101\}$ represents $C(4)$ and the corresponding set of permutations is $\{i d ;(12) ;(23) ;(34) ;(12)(34)\}$ in cycle notation, i.e.

$$
\{1234 ; 2134 ; 1324 ; 1243 ; 2143\} \text {. }
$$

However, we will see very soon that the lower bound in the last Corollary can be improved. The asymptotic improvement we obtain will be a direct consequence of the following inequality that follows easily from Proposition 4.1.

Proposition 4.5. For every $n \in \mathbb{N}$ we have:

$$
\rho(L) \geq \log \sqrt[n]{\rho(L, n)} .
$$

Proof. By Proposition 4.1 we have $\sqrt[n k]{\rho(L, n k)} \geq \sqrt[n]{\rho(L, n)}$.

This justifies our interest in calculating $\rho(L, n)$ for the first values of $n$. The results are shown in the following table. 


\begin{tabular}{|c|c|c|c|c|c|c|c|}
\hline$n$ & 1 & 2 & 3 & 4 & 5 & 6 & 7 \\
\hline$\rho(L, n)$ & 1 & 2 & 3 & 6 & 10 & 20 & 35 \\
\hline
\end{tabular}

For $n=7$ we built a clique of size 35 by putting together 7 cliques each of size 5 , obtained as cyclic shifts of certain sequences of length 5. Before explaining this construction in more detail, we prove a general result on cyclic shifts for any graph $G$.

Let $A \subseteq \mathbb{N}$ with $|A|=k$. Let $\pi=a_{1} \ldots a_{k}$ be an arrangement of $A=\left\{a_{1}, \ldots, a_{k}\right\}$ on a cycle of length $k$ : we say that $\pi$ is a circular arrangement of $A$. We define the circular distance $\partial_{\pi}\left(a_{i}, a_{j}\right)$ of $a_{i}$ and $a_{j}$ with respect to $\pi$ as follows:

$$
\partial_{\pi}\left(a_{i}, a_{j}\right)=\left\{\begin{array}{cc}
0 & \text { if } i=j \\
\min \{j-i, k+i-j\} & \text { if } i<j \\
\partial_{\pi}\left(a_{j}, a_{i}\right) & \text { if } i>j .
\end{array}\right.
$$

We say that a circular arrangement $\pi=a_{1} \ldots a_{k}$ is complete if for every $d=$ $1, \ldots,\left\lfloor\frac{k}{2}\right\rfloor$ there exists an edge $\left\{a_{i}, a_{j}\right\} \in E(G)$ with $\partial_{\pi}\left(a_{i}, a_{j}\right)=d$.

Lemma 4.6. If $\pi=a_{1} \ldots a_{k}$ is a complete circular arrangement of $A$, then the subset $S(\pi)$ of $R(A)$ consisting of all the cyclic shifts of $\pi$, i.e.

$$
S(\pi)=\left\{\pi^{d}=a_{d} a_{d+1} \ldots a_{d+(k-1)}: d=1, \ldots, k\right\}
$$

where

is a clique in $G(A)$.

$$
a_{r}=a_{s} \Leftrightarrow r \equiv s(\bmod k)
$$

Proof. It is enough to show that for any $t=2, \ldots k$ one has $\left\{\pi, \pi^{t}\right\} \in E(G(A))$. First start with any $t$ such that $t \leq\left\lfloor\frac{k}{2}\right\rfloor$ : since $\pi$ is complete, there exists $\left\{a_{i}, a_{j}\right\} \in$ $E(G)$ such that $\partial_{\pi}\left(a_{i}, a_{j}\right)=t$ : we can fix $i<j$. If the circular distance $t$ is achieved as $j-i$, then $\left\{\pi, \pi^{t}\right\} \in E(G(A))$ since in coordinate $i$ one has $\left\{\pi_{i}, \pi_{i}^{t}\right\}=$ $\left\{a_{i}, a_{j}\right\} \in E(G)$, and also $\left\{\pi, \pi^{k-t+1}\right\} \in E(G(A))$ since in coordinate $j$ one has $\left\{\pi_{j}, \pi_{j}^{k-t+1}\right\}=\left\{a_{j}, a_{i}\right\} \in E(G)$; if the circular distance $t$ is achieved as $k+i-j$, then $\left\{\pi_{j}, \pi_{j}^{t}\right\}=\left\{a_{j}, a_{i}\right\}$ and $\left\{\pi_{i}, \pi_{i}^{k-t+1}\right\}=\left\{a_{i}, a_{j}\right\}$. In any case both $\left\{\pi, \pi^{t}\right\}$ and $\left\{\pi, \pi^{k-t+1}\right\}$ are edges of $G(A)$; consequently $\left\{\pi, \pi^{t}\right\} \in E(G(A))$ for $t=2, \ldots k$.

\section{Proposition 4.7.}

$$
\rho(L, 7)=35 .
$$

Proof. Let $\Gamma$ be the set consisting of the following sequences:

$$
\begin{aligned}
& \alpha_{1}^{\prime}=23546 \\
& \alpha_{1}^{\prime \prime}=32546 \\
& \alpha_{2}^{\prime}=23547 \\
& \alpha_{2}^{\prime \prime}=54237 \\
& \beta_{1}^{\prime}=34651 \\
& \beta_{1}^{\prime \prime}=65341 \\
& \beta_{2}=14357 .
\end{aligned}
$$

Each sequence in $\Gamma$ is a complete circular arrangement of the corresponding set of its entries. By Lemma 4.6 it follows that $S(\gamma)$ is a clique, for any $\gamma \in \Gamma$.

First we show that $A_{1}:=S\left(\alpha_{1}^{\prime}\right) \cup S\left(\alpha_{1}^{\prime \prime}\right)$ is a clique in $L(\{2,3,4,5,6\})$. 


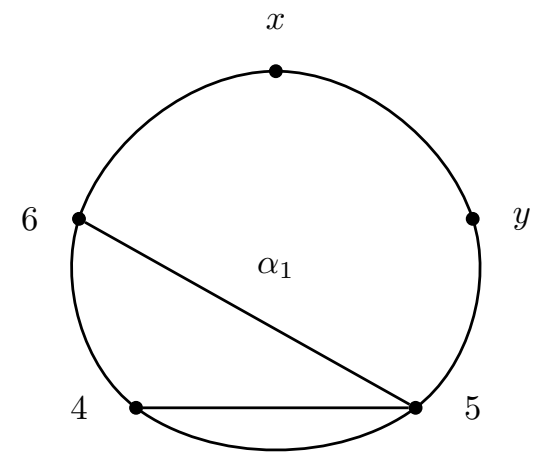

Observe that any sequence of the form $x y 546$ is already a complete circular arrangement of $\{x, y, 4,5,6\}$ (we are not using the edges $\{2,3\}$ and $\{3,4\}$ to achieve the adjacencies): the same argument as in Lemma 4.6 makes clear that $\left(\alpha_{1}^{\prime}\right)^{d}$ is adjacent to any element of $S\left(\alpha_{1}^{\prime \prime}\right)$ of the form $\left(\alpha_{1}^{\prime \prime}\right)^{t}$ with $t \neq d$, while when $t=d$ then $\left(\alpha_{1}^{\prime}\right)^{d}$ and $\left(\alpha_{1}^{\prime \prime}\right)^{d}$ will be colliding in coordinate $d$, because $\left(\alpha_{1}^{\prime \prime}\right)^{d}$ is obtained from $\left(\alpha_{1}^{\prime}\right)^{d}$ by interchanging 2 and 3 .

Now we show that $A_{2}:=S\left(\alpha_{2}^{\prime}\right) \cup S\left(\alpha_{2}^{\prime \prime}\right)$ is a clique in $L(\{2,3,4,5,7\})$.

Notice that we have $\alpha_{2}^{\prime \prime}=\phi \circ \alpha_{2}^{\prime}$, with $\phi=(2,5) \circ(3,4)$, where $(i, j)$ is the transposition of $i$ and $j$. Clearly $\left\{\alpha_{2}^{\prime}, \alpha_{2}^{\prime \prime}\right\} \in E(L(\{2,3,4,5,7\}))$ : they collide in the second coordinate through the edge $\{3,4\}$. Observe that we can find two 2-sets $\{a, b\}$ (precisely $\{3,5\}$ and $\{2,4\}$ ) at circular distance respectively 1 and 2 in $\alpha_{2}^{\prime}$, such that the corresponding 2-sets of the form $\{a, \phi(b)\}$ (precisely $\{3,2\}$ and $\{2,3\}$ ) are edges of $L$ : hence the same reasoning as in Lemma 4.6 can be applied to establish that we also have $\left\{\alpha_{2}^{\prime},\left(\alpha_{2}^{\prime \prime}\right)^{d}\right\} \in E(L(\{2,3,4,5,7\}))$ for $1<d \leq 5$.

As for $B_{1}:=S\left(\beta_{1}^{\prime}\right) \cup S\left(\beta_{1}^{\prime \prime}\right)$, we notice that the bijection

$$
\phi=\left(\begin{array}{lllll}
2 & 3 & 4 & 5 & 7 \\
3 & 4 & 5 & 6 & 1
\end{array}\right)
$$

is an isomorphism of $L(\{2,3,4,5,7\})$ into $L(\{1,3,4,5,6\})$ such that $\beta_{1}^{\prime}=\phi \circ \alpha_{2}^{\prime}$ and $\beta_{1}^{\prime \prime}=\phi \circ \alpha_{2}^{\prime \prime}$ : so the argument used for the set $A_{2}$ applies to $B_{1}$ as well, showing that the latter is a clique in $L(\{1,3,4,5,6\})$.

Now let $s X:=\{s \mathbf{x}: \mathbf{x} \in X\}$ (resp. $X s$ ) be the set of sequences obtained from those in $X$ by prefixing (resp. postfixing) to each of them the symbol $s$ and set

$$
\begin{gathered}
A=1 A_{1} 7 \cup 1 A_{2} 6, \\
B=2 B_{1} 7 \cup 2 S\left(\beta_{2}\right) 6 .
\end{gathered}
$$

The sets $A$ and $B$ are both cliques in $L(7)$ since the adjacency between elements of $1 A_{1} 7$ and $1 A_{2} 6$, or between those of $2 B_{1} 7$ and $2 S\left(\beta_{2}\right) 6$, is guaranteed in the last coordinate, where we use the edge $\{6,7\}$. Finally $C=A \cup B$ is a clique in $L(7)$, since the adjacency between elements of $A$ and $B$ is established in the first coordinate, where we use the edge $\{1,2\}$. Obviously, $C$ has 35 elements.

Conjecture. Encouraged by the previous clique of size 35 we are tempted to formulate the following conjecture:

$$
\rho(L, n)=\left(\begin{array}{c}
n \\
\left\lfloor\frac{n}{2}\right\rfloor
\end{array}\right) .
$$


Unfortunately, we do not have more serious reasons to believe in it.

Now we are ready to improve the lower bound $\rho(L) \geq \log _{2}\left(\frac{1+\sqrt{5}}{2}\right)=0.6942 \ldots$ of Proposition 4.4.

\section{Proposition 4.8.}

$$
\rho(L) \geq 0.732 \ldots
$$

Proof. Combining the statement of Proposition 4.5 with that of the last Proposition 4.7 we immediately see that $\rho(L, n) \geq 35^{\frac{n}{7}}$, and $\rho(L) \geq \frac{1}{7} \log _{2} 35=0.732 \ldots$.

\section{SURPRISE CAPACITY}

Let $G$ be once again an arbitrary graph with countable vertex set $\mathbb{N}$ and let $G^{n}$ be the same power graph as before. We will say that the set $C \subseteq \mathbb{N}^{n}$ generates a surprise clique in $G^{n}$ if $C$ generates a clique in $G^{n}$ with the property that for any $\{\mathbf{x}, \mathbf{y}\} \in\left(\begin{array}{c}\mathbb{N}^{n} \\ 2\end{array}\right)$ the ordered pairs of coordinates $\left(x_{i}, y_{i}\right)$ are all different. We shall denote by $S(G, n)$ the maximum cardinality of a surprise clique in $G^{n}$ and will call the limit

$$
\limsup _{n \rightarrow \infty} \frac{1}{n} \log _{2} S(G, n)
$$

the surprise capacity of $G$. Clearly, this quantity is lower bounded by the permutation capacity of the same graph. (At the end of the paper we will comment on the intuitive meaning of this definition.)

Proposition 5.1. The maximum cardinality of a surprise clique in $L^{n}$ is $2^{n}$, where $L$ is the infinite path.

Proof. We shall use the easy and well-known sub-multiplicativity of the chromatic number, i.e. $\chi\left(G^{n}\right) \leq[\chi(G)]^{n}$. To verify this, let $c: V(G) \rightarrow \mathbb{N}$ be an optimal coloring of $G$. Then the map $c^{n}:[V(G)]^{n} \rightarrow \mathbb{N}^{n}$ defined by $c^{n}\left(x_{1} \ldots x_{n}\right)=c\left(x_{1}\right) \ldots c\left(x_{n}\right)$ is a proper coloring of $G^{n}$. The chromatic number of the infinite path $L$ is 2: so for the power graph $L^{n}$ one has $\chi\left(L^{n}\right)=2^{n}$ and consequently $\omega\left(L^{n}\right) \leq 2^{n}$. It follows that if $C$ is a surprise clique in $L^{n}$, then

$$
|C| \leq 2^{n},
$$

since any surprise clique is in particular a clique.

We now construct a clique of cardinality $2^{n}$, showing that

$$
S(L, n) \geq 2^{n} .
$$

Our construction will consist of appropriately chosen sequences of length $n$, with entries from $[2 n]$, where lack of repetition will be ensured by strict monotonicity. For any binary sequence $\mathbf{x}$ of length $n$, define

$$
a(\mathbf{x}):=x_{1}, x_{1}+x_{2}, \ldots, \sum_{j=1}^{n} x_{j},
$$

that is

$$
a(\mathbf{x})_{i}:=\sum_{j=1}^{i} x_{j} .
$$


Apply this to binary strings on the alphabet $\{1,2\}$ and set

$$
C:=\left\{a(\mathbf{x}): \mathbf{x} \in\{1,2\}^{n}\right\} \subseteq[2 n]^{n} .
$$

By construction one has $a(\mathbf{x}) \neq a\left(\mathbf{x}^{\prime}\right)$ if and only if $\mathbf{x} \neq \mathbf{x}^{\prime}$. Hence $C$ has the same cardinality as the set of all the binary sequences of length $n$. Now we show that $C$ is a clique in the $n$-th power of $L$. Take $\mathbf{x}, \mathbf{x}^{\prime} \in\{1,2\}^{n}$ with $\mathbf{x} \neq \mathbf{x}^{\prime}$ and let $s$ be the first coordinate in which the two binary sequences differ: then for the corresponding sequences in $C$ we have $\left\{a(\mathbf{x}), a\left(\mathbf{x}^{\prime}\right)\right\} \in E\left(L^{n}\right)$, since $\left|a(\mathbf{x})_{s}-a\left(\mathbf{x}^{\prime}\right)_{s}\right|=\mid \sum_{j=1}^{s} x_{j}-$ $\sum_{j=1}^{s} x_{j}^{\prime}|=| x_{s}-x_{s}^{\prime} \mid=1$. Finally the condition for $C$ to be a surprise clique holds, since by the definition (2) its elements are strictly increasing sequences, hence there are no repetitions of symbols.

The above proposition shows that the surprise capacity of the infinite path is 1 .

\section{UNIMODAL PERMUTATIONS}

Let us return to $L(n)$, the graph induced in the power graph $L^{n}$ by the set of all permutations of $[n]$. In order to see the wealth of relatively large cliques in $L(n)$ it might be interesting to understand the density of cliques in the relatively small set of unimodal permutations.

We say that a permutation a of $[n]$ is unimodal if there is an index $h \in[n]$ such that $a_{1}<a_{2}<\ldots<a_{h}>a_{h+1}>\ldots>a_{n}$. We will introduce a new variant of our introductory problem of determining the number $\rho(n)$ of the maximum cardinality of a set of pairwise colliding permutations of $n$ (recall that this concept was at the core of our permutation capacity problem).

Let us denote by $U(n)$ the maximum cardinality of a set of pairwise colliding unimodal permutations of $n$. We claim

\section{Theorem 6.1.}

$$
\log _{2} \frac{1+\sqrt{5}}{2} \leq \limsup _{n \rightarrow \infty} \frac{1}{n} \log _{2} U(n) \leq 1
$$

Proof. The upper bound is an obvious consequence of Proposition 4.2, since $U(n) \leq \rho(L, n)$.

Fix $\alpha \in(0,1)$ and $\beta \in(0,1)$ in a way to be specified later. Write $l_{n}=\lfloor\alpha n\rfloor$ and $k_{n}=\lfloor\beta \alpha n\rfloor$. Now we shall adapt the construction in the proof of Proposition 5.1 to define our unimodal permutations. To this purpose consider the set $B_{n}$ of all the sequences in $\{1,2\}^{l_{n}}$ in which the symbol 2 appears $k_{n}$ times. Thus by the well-known asymptotics of the binomial coefficients (Lemma 2.3 p.30 of [4])

$$
\left|B_{n}\right|=\left(\begin{array}{l}
l_{n} \\
k_{n}
\end{array}\right) \geq \frac{1}{n+1} 2^{l_{n} h\left(k_{n} / l_{n}\right)},
$$

where $h(t)=-t \log _{2} t-(1-t) \log _{2}(1-t)$ is the binary entropy function.

To every $\mathbf{x} \in B_{n}$ we shall associate as before the increasing sequence of natural numbers $a(\mathbf{x})$ whose $i$-th element $a_{i}(\mathbf{x})$ is as in formula (2). Suffixing to the sequence $a(\mathbf{x})$ the naturals from $[n] \backslash\left\{a_{1}(\mathbf{x}), \ldots a_{l_{n}}(\mathbf{x})\right\}$ in decreasing order we obtain the unimodal sequence $\widehat{\mathbf{x}}$ of integers from the set $\left[l_{n}+k_{n}\right]$, where 
$l_{n}+k_{n}=\lfloor\alpha n\rfloor+\lfloor\beta \alpha n\rfloor$. Then in order for $\widehat{\mathbf{x}}$ to be a permutation of $[n]$, we must have $\alpha(1+\beta) \leq 1$, i.e.

$$
\alpha \leq \frac{1}{1+\beta} .
$$

Clearly the relation between $\mathbf{x}$ and $\widehat{\mathbf{x}}$ is bijective and therefore we have obtained $\left|B_{n}\right|$ unimodal permutations. As in Proposition 5.1, we have that if $\mathbf{x} \neq \mathbf{x}^{\prime}$, then $a(\mathbf{x})$ and $a\left(\mathbf{x}^{\prime}\right)$ are colliding: so the corresponding sequences $\widehat{\mathbf{x}}$ and $\widehat{\mathbf{x}^{\prime}}$ will be colliding too.

We just saw that the set $\widehat{B_{n}}=\left\{\widehat{\mathbf{x}}: \mathbf{x} \in B_{n}\right\}$ has the same cardinality as $B_{n}$ and it is a clique of unimodal elements of $L(n)$.

Recalling the definition of $U(n)$ and (3), we deduce that

$$
U(n) \geq \frac{1}{n+1} 2^{l_{n} h\left(k_{n} / l_{n}\right)}
$$

whence

$$
\limsup _{n \rightarrow \infty} \frac{1}{n} \log _{2} U(n) \geq \limsup _{n \rightarrow \infty} \frac{l_{n}}{n} h\left(k_{n} / l_{n}\right)=\alpha h(\beta) .
$$

Choosing the largest $\alpha$ with respect to the constraint in (4), i.e. $\alpha=\frac{1}{1+\beta}$, and maximizing in $\beta$ we obtain:

$$
\limsup _{n \rightarrow \infty} \frac{1}{n} \log _{2} U(n) \geq \max _{\beta \in(0,1)} \frac{h(\beta)}{1+\beta}=\log _{2} \frac{1+\sqrt{5}}{2} .
$$

In order to see that our last entropy expression has as its maximum the logarithm of the golden ratio, as claimed, the reader is referred to [8].

It is tempting to believe the lower bound to be tight, even though we have no real reason to do so.

\section{Concluding REMARKS}

In this paper we have introduced several closely related concepts of capacity for infinite graphs. It is not clear whether these can have the same interpretation in terms of Shannon's theory of information as do the concepts of Shannon capacity [13] and Sperner capacity [6]. In particular, the Shannon capacity of a finite simple graph is the highest rate at which one can transmit data over a discrete memoryless (stationary) channel with zero probability of error. Recently Nayak and Rose [12] showed that Sperner capacity is the key in determining the analogous transmission rate for compound channels with an uninformed coder-decoder pair.

The common feature of our models is that for no codeword pairs can we transmit the same symbol pair at different instants of time. This restriction might be of relevance if one is to guarantee security of transmission; an intruder can never experience the repetition of a symbol configuration and thereby learn how to adapt to a hitherto unknown communication situation it creates.

Having a disposable symbol set does not necessarily mean that the channel has an infinite input alphabet. In fact note that in all our code constructions every symbol has at most seven different "successors".

Acknowledgements. We would like to thank Miki Simonovits for his friendly interest. 


\section{REFERENCES}

[1] P.J. Cameron and C.Y. Ku, "Intersecting families of permutations", European J. Combin. 2 n.7 (2003) 881-890.

[2] G. Cohen, J. Körner and G. Simonyi, "Zero-error capacities and very different sequences" in Sequences, combinatorics, compression, security and transmission R.Capocelli ed., SpringerVerlag (1990) 87-101.

[3] I. Csiszár and J. Körner, "On the capacity of the arbitrarily varying channel for maximum probability error", Z. Wahrscheinlichkeittheorie verw. Geb. 57 (1981) 87-101.

[4] I. Csiszár and J. Körner, Information Theory: Coding Theorems for Discrete Memoryless Systems, Academic Press, New York (1982) and Akadémiai Kiadó, Budapest (1981) (460 pp).

[5] A. Galluccio, L. Gargano, J. Körner and G. Simonyi, "Different capacities of digraphs", Graphs and Combinatorics, 10(1994), pp. 105-121.

[6] L. Gargano, J. Körner and U. Vaccaro, "Sperner Capacities", Graphs and Combinatorics, 9 (1993), 31-46

[7] L. Gargano, J. Körner and U. Vaccaro, "Capacities: from information theory to extremal set theory", J.Combin.Theory Ser.A 68 (1994) 296-316.

[8] J. Körner and G. Simonyi, "A Sperner-Type Theorem and Qualitative Independence", J.Combin.Theory Ser.A 59n.1 (1992) 90-103.

[9] C.Y. Ku and I. Leader, "An Erdős-Ko-Rado Theorem for Partial Permutations", submitted to Discrete Math.

[10] B. Larose and C. Malvenuto, "Stable sets of maximal size in Kneser-type graphs", European J. Combin. 25 n.5 (2004) 657-673.

[11] L. Lovász, "On the Shannon capacity of a graph", IEEE Trans. Inform. Theory 25 (1979) $1-7$.

[12] J. Nayak and K. Rose, "Graph capacities and zero-error transmission over compound channels", IEEE Trans. Inform. Theory, to appear.

[13] C.E. Shannon, "The zero-error capacity of a noisy channel" IRE Trans. Inform. Theory 2 (1956) 8-19.

[14] J.H. Van Lint and R.M. Wilson, A course in Combinatorics, Cambridge University Press, Cambridge (1992).

(János Körner) Dipartimento di Informatica, Università di Roma "La Sapienza", Via SALARIa 113, 00198 Roma, ItALIA

E-mail address: korner@di.uniroma1.it

(Claudia Malvenuto) Dipartimento di Informatica, Università di Roma "La Sapienza", Via Salaria 113, 00198 Roma, Italia

E-mail address: claudia@di.uniroma1.it 